\title{
Comparison of Chromogenic Culture Media, Rapid Immunochromatographic Test and Temocillin Resistance for The Detection of OXA-48 Carbapenemase-Positive Klebsiella Pneumonia Strains
}

\author{
Erkan Sanmak ${ }^{1 *}$, Sebahat Aksaray ${ }^{2}$
}

${ }^{1}$ Department of Medical Microbiology, Mardin State Hospital Medical Microbiology Laboratory, Mardin, Turkey

${ }^{2}$ Department of Health Sciences,

Haydarpasa Numune Training And Research Hospital, Istanbul, Turkey

Correspondence:

Erkan Sanmak

Address: Department of Medical Microbiology, Mardin State Hospital Medical Microbiology Laboratory, Mardin, Turkey

Email: erkansanmak1@gmail.com

Received: 09.04.2021,

Accepted: 07.09.2021

https://doi.org/10.29333/jcei/11267

\begin{abstract}
Objectives: The study was aimed to compare the performance of three different approaches (temocillin disc diffusion test (TDD), immunochromatographic assay, OXA-48 K-SET, and chromogenic assay containing ChromID OXA-48 media) with PCR in a collection of $K$. pneumoniae producing OXA-48.
\end{abstract}

Materials and methods: $A$ total of 45 carbapenem resistant $K$. pneumoniae isolates (27 were OXA-48 positive) were included in the study. Polymerase chain reaction (PCR), TDD, OXA-48 K-SET, ChromID OXA-48 media were applied to all isolates.

Results: The OXA-48 K-SET was found to be $100 \%$ compatible with gold standard PCR for the identification of OXA-48 positive Klebsiella pneumoniae strains. $100 \%$ sensitivity and $88.8 \%$ specificity were found with the ChromID OXA-48 medium method. Sensitivity and specificity of the TDD assay were found $100 \%$ and $77.8 \%$, respectively.

Conclusion: The OXA-48 K-Set had excellent performance for detecting OXA-48 carbapenemase and can be used easily and reliably in the routine clinical microbiology laboratory for rapid detection of OXA-48 producing $\mathrm{K}$. pneumoniae isolates, particularly in endemic regions. ChromID media and temocillin disc diffusion testing can be applied in laboratories as part of the identification and screening of carbapenemase-positive Klebsiella pneumoniae.

Keywords: carbapenemase, ChromID, K-set, Klebsiella pneumoniae, OXA-48

\section{INTRODUCTION}

Klebsiella pneumoniae (K. pneumoniae) is an important pathogen that is responsible for various types of infections in humans, including pneumonia, septicemia, urinary tract infections, in both community and hospital settings [1]. Carbapenems are thought to be the last-line treatment of $\mathrm{K}$. pneumoniae infections, and resistance to them is a serious problem for infection control worldwide [2]. Antibiotic-resistant microorganisms can lead to severe infections with high morbidity and mortality, as well as increasing length of hospital stay and cost of treatment [3]. Rapid spread of carbapenemresistant $\mathrm{K}$. pneumoniae ( $\mathrm{CR}-\mathrm{Kp}$ ) has been reported in various healthcare facilities around the world in the last decade [4]. The major mechanism of carbapenem resistance in K. pneumoniae has been associated with the production of carbapenem hydrolyzing enzymes including Klebsiella pneumoniae carbapenemase (KPC), New Delhi metallob-lactamase (NDM), and oxacillinase (OXA) type carbapenemase [5]. The OXA-48 carbapenemase is an Ambler class $D \beta$ lactamase with carbapenem hydrolyzing oxacillinase activity and was identified from sporadic CR-Kp isolates from Turkey in 2001 [6]. Since then it has dramatically increased in Europe, India and Africa in last two decades. The rapid and accurate identification of OXA-48 type carbapenemase is crucial to ensure timely implementation of infection control measures. Standard detection of OXA-48 is 
based on molecular techniques, mostly polymerase chain reaction (PCR), which is costly and difficult to apply for many laboratories [7]. Different non-molecular techniques have been used to detect carbapenem resistant bacteria, including phenotypic tests (combination disk test, gradient diffusion strips, double disk synergy test), colorimetric tests based on carbapenem hydrolysis (cloverleaf method, colorimetric assays, Carba NP, Blue-CARBA, carbapenem inhibition, starch-iodine assay) and immunochromatographic methods [8,9]. However, the results of studies evaluating OXA-48-producing bacteria using non-molecular techniques are inconclusive and limited.

The aim of this study was to compare the performance of three different approaches (temocillin disc diffusion susceptibility testing-a new immunochromatographic assay, OXA-48 K-SET, and chromogenic assay containing ChromID agar) with PCR in a collection of K. pneumonia isolates producing OXA-48.

\section{METHOD}

A total of 45 non-duplicated carbapenem resistant $\mathrm{K}$. pneumoniae isolates from various clinical specimens were obtained from patients treated in Haydarpasa Numune Training and Research Hospital, Istanbul, Turkey. Urine, tracheal aspirate, sputum and wound samples were incubated for $18-24$ hours at $37^{\circ} \mathrm{C}$ on $5 \%$ sheep blood agar and MacConkey agar. Blood culture vials were incubated in an automated system (BacT Alert 3D, BioMerieux, France) for 7 days. After positive signals were obtained, gram staining was inoculated into appropriate medium as described above. The VITEK2 Compact system (bioMérieux, Marcyl'Etoile, France) was used for the identification of the isolates and antibiotic susceptibility testing. Confirmation of carbapenem resistance was determined by disk diffusion method according to Clinical and Laboratory Standards Institute guidelines. All isolates with decreased susceptibility or resistance to at least one carbapenem were considered as resistant. Strains were stored in duplicate in $-20^{\circ} \mathrm{C}$ in trypticase soy broth with glycerol $16 \%$. All isolates showing evidence of carbapenem resistance by disk diffusion and VITEK2 system were investigated using real-time PCR (RT-PCR) assays to determine whether the resistance was due to the OXA-48 gene. The PCR amplification and sequencing of the blaoxA-48 gene was performed on Rotor-Gene 6000 instrument with Qiagen Artus RG PCR kit (Qiagen, Hamburg, Deutschland).

\section{Temocillin Disc Diffusion}

To investigate temocillin sensitivity, carbapenemase producing $\mathrm{K}$. pneumoniae strains were inoculated on Mueller Hinton II agar plates (BioMérieux, France) with 30 $\mu \mathrm{g}$ temocillin discs (Bioanalyse, Turkey), using a 0.5 McFarland bacterial suspension. It was evaluated after 18-24 hours of incubation at $35-37^{\circ} \mathrm{C}$. The presence of a $\leq 10 \mathrm{~mm}$ zone with the temporal disc was considered as phenotypic marker for OXA-48 carbapenemase positivity.

\section{Rapid Diagnostic Test for OXA-48}

All isolates were evaluated with OXA-48 K-SeT assay kits (Coris BioConcept, Gembloux, Belgium) according to the manufacturer's instructions. Briefly, a single colony was resuspended in 10 drops of lysis buffer (Tris- $\mathrm{HCl}, \mathrm{NaN} 3$ [pH 7.5]) for protein extraction, and 3 drops of this extracted homogenized solution was then applied to the sample well. The results were assessed within 15 minutes at room temperature with naked eye.

\section{Chromogenic Media}

ChromID OXA-48 agar was supplied from Biomerieux, France as a ready-for-use plate medium. Carbapenem resistant $\mathrm{K}$. pneumoniae isolates were inoculated on ChromID OXA-48 agar and incubated for 18-24 hours at 35$37^{\circ} \mathrm{C}$ in an aerobic environment. According to the manufacturer's recommendation, green colonies were identified to be positive for OXA-48-producing $\mathrm{K}$. pneumoniae.

\section{Statistical Analysis}

Statistical Analyses were performed with SPSS v20.0 (SPSS Inc., Chicago, IL, USA). The performance of different testing methods were evaluated by using PCR as the gold standard. The test performance parameters were evaluated by sensitivity, specificity, positive predictive value (PPV), negative predictive value (NPV), and overall accuracy. The sensitivity of the tests was calculated from the number of correctly determined OXA-48 and K. pneumaniae producing organisms, while the specificity was calculated from the number of true-negative isolates.

\section{RESULTS}

Forty-five K. pneumoniae isolates resistant to at least one carbapenem antibiotic were included the study. $\mathrm{K}$. pneumoniae isolates were investigated in terms of OXA resistance genes via RT-PCR (Figure 1). The OXA-48 gene was detected in 27 (60\%) of the 45 isolates.

All of the OXA-48 positive isolates were found to be temocillin resistant. Fourteen of the 18 strains in which the OXA-48 gene was not detected via PCR were found to be susceptible in the temocillin disk diffusion test. The remaining 4 isolates (without the OXA-48 gene) were found to be resistant in the disk diffusion test. The sensitivity was found to be $100 \%$ and the specificity was $77.8 \%$, with the PCR test defined as the gold standard. The PPV of the test was $87 \%$ and the NPV was $100 \%$. The total accuracy of the test was determined as 91\% (Table 1, Figure 2).

The OXA-48 K-SeT assay gave a positive result for all 27 OXA-48-producing strains and was negative for all of the other isolates tested. False positives or false negatives were not detected. Therefore, the sensitivity and specificity of the OXA-48 K-SeT test were determined as $100 \%$. The PPV and 


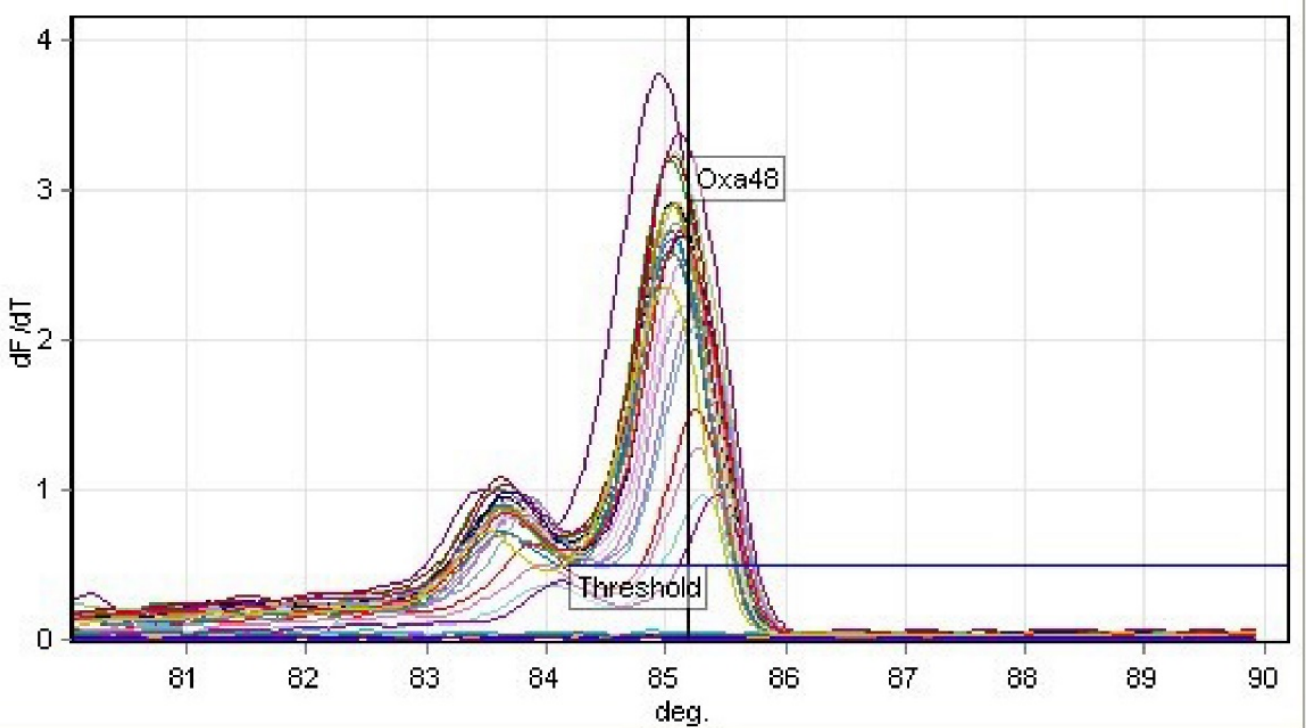

Figure 1. Evaluation of OXA 48 gene using RT-PCR

Table 1. The comparison of PCR and temocillin disc diffusion testing

\begin{tabular}{ccccc}
\hline & & & Polymerase chain reaction \\
\hline \multirow{3}{*}{ Temocillin disc diffusion } & & Positive & Negative & \multicolumn{1}{c}{ Total } \\
\cline { 2 - 5 } & Positive & 27 & 4 & 31 \\
\cline { 2 - 5 } & Negative & 0 & 14 & 14 \\
\cline { 2 - 5 } & Total & 27 & 18 & 45
\end{tabular}

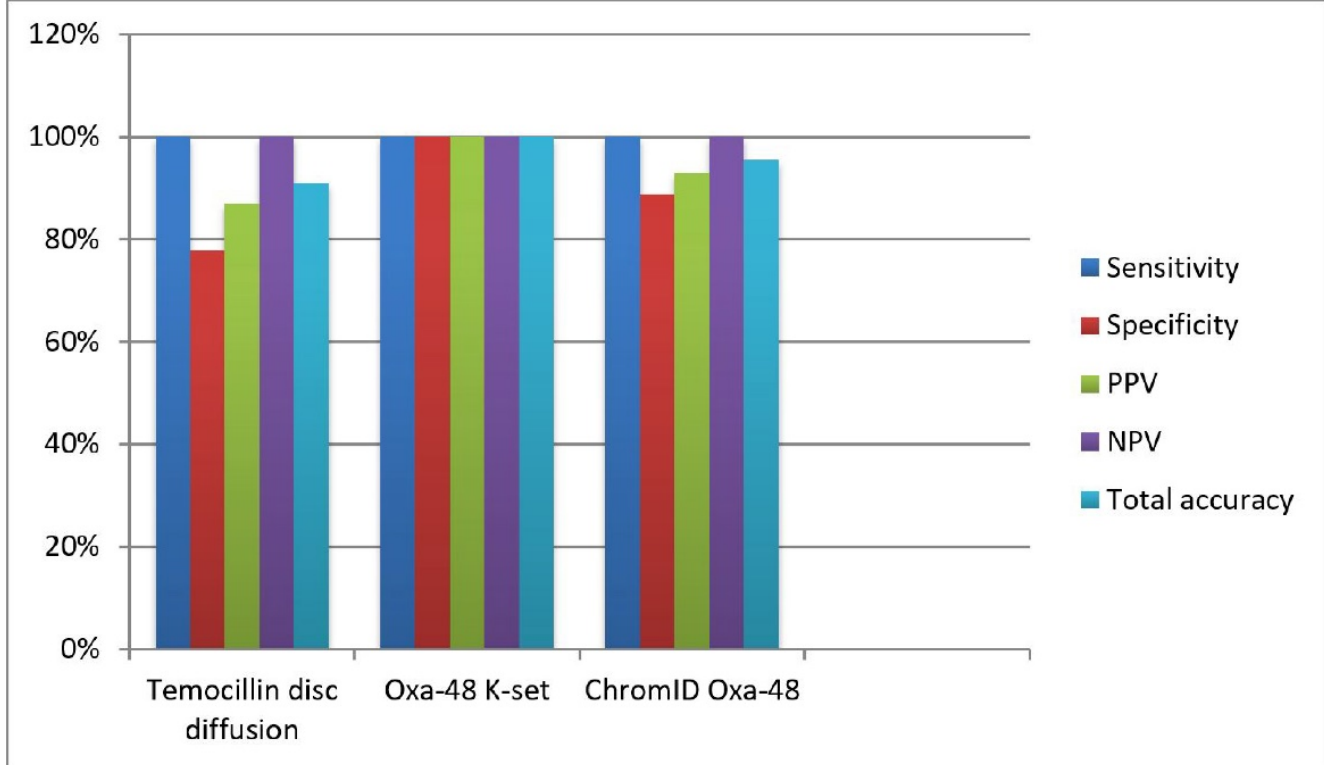

Figure 2. The comparison of performance results of the different testing methods with PCR

PCR: Polymerase chain reaction, PPV: Positive predictive value, NPV: Negative predictive value

NPV of the test was $100 \%$ and the total accuracy of the test was also calculated as 100\% (Table 2, Figure 2).

All Oxa-48 positive K. pneumoniae isolates formed green colonies on ChromID Oxa-48 agar. However, two strains negative for the OXA-48 gene in PCR had growth on the ChromID OXA-48 agar. The sensitivity was calculated as $100 \%$ and the specificity as $88.8 \%$, according to gold standard
RT-PCR. The PPV of the test was found to be $93.1 \%$ and the NPV was $100 \%$. The overall accuracy of the test was $95.5 \%$ (Table 3, Figure 2).

\section{DISCUSSION}

This study was aimed at investigating sensitivity and specificity of the temocillin disc diffusion test, the 
Table 2. The comparison of PCR and Oxa-48 K-Set testing

\begin{tabular}{ccccc}
\hline & & \multicolumn{3}{c}{ Polymerase chain reaction } \\
\hline & & Positive & Negative & Total \\
\hline \multirow{2}{*}{$\begin{array}{c}\text { Oxa-48 } \\
\text { K-SET test }\end{array}$} & Positive & 27 & 0 & 27 \\
\cline { 2 - 5 } & Negative & 0 & 18 & 18 \\
\cline { 2 - 5 }
\end{tabular}

Table 3. The comparison of PCR and ChromID Oxa-48 testing

\begin{tabular}{lcccc}
\hline & & & Polymerase chain reaction \\
\hline \multirow{3}{*}{ ChromID Oxa-48 test } & & Positive & Negative & Total \\
\cline { 2 - 5 } & Positive & 27 & 2 & 29 \\
\cline { 2 - 5 } & Negative & 0 & 16 & 16 \\
\hline
\end{tabular}

immunochromatographic assay (OXA-48 K-set rapid test) and the chromogenic test containing ChromID OXA-48 agar for the detection of OXA-48 producing $\mathrm{K}$. pneumoniae in a local setting. We demonstrated that the OXA-48 K-set rapid test was highly sensitive and specific for the confirmation of OXA-48-like carbapenemase within 15 minutes. The temocillin disc diffusion test and the ChromID OXA-48 agar demonstrated a lower level of accuracy and may not be sufficient to accurately identify the presence of OXA-48.

The global spread of nosocomial infections due to CR-Kp is a major public health concern, particularly in newborns, those with malignancy and immunocompromised patients in regions with high prevalence of resistance [10]. Accurate and rapid identification is at the basis of swift treatment and control of these highly resistant pathogens, and also the implementation of appropriate infection control measures to prevent further dissemination. KPCs were first isolated in 1996 in the USA [11]. Since 1996, carbapenemase genes have been spread globally in the Enterobacteriaceae family, particularly K. pneumoniae. The European carbapenemaseproducing Enterobacteriaceae (CPE) Survey initiative study showed in 2017 that the infection prevalence of CPE was 1.3 per 10,000 hospital admissions [12]. In a study evaluating $2301 \mathrm{~K}$. pneumonia isolates, it was found that $37 \%$ of carbapenem-resistant $K$. pneumoniae strains had a carbapenemase gene, with genes encoding KPC (42\%) and OXA-48 (38\%) carbapenemases determined as the most common types [12]. Turkey, Italy and Greece were deemed as having the highest epidemiologic level (stage 5 "endemic situation") of these CPE isolates in two different epidemiological studies conducted in 2014-2015 and in 2018 $[13,14]$. The main mechanism of carbapenemase resistance is hydrolysis of carbapenems by carbapenemase enzymes, including KPC and OXA-48, which are encoded mainly on plasmids and are highly transmissible. The OXA-48 carbapenemase has $\beta$-lactamase activity with carbapenem hydrolyzing oxacillinase and was first demonstrated in 2001 from a Klebsiella pneumoniae isolate in Turkey [6]. Recent epidemiological studies have reported that OXA-48 carbapenemase is the most prevalent carbapenemase in European and Middle-Eastern countries [15], with a particular emphasis in Turkey. Davarc1 et al. showed in a recent molecular epidemiology study that 45 (\%3.1) of the $1452 \mathrm{~K}$. pneumoniae isolates were carbapenem-resistant and 32 (\%71.1) of these were OXA-48 gene positive [16]. We investigated the OXA-48 resistance gene in CR-Kp strains via $\mathrm{RT}-\mathrm{PCR}$ and found that $60 \%$ of the strains were positive. Compared to other studies, the percentage of resistance in our study was higher. In limited studies on this subject in Turkey, resistance frequency is generally shown to be higher compared to other countries. This is an expected result because the OXA-48 resistance gene was first isolated in Turkey and the presence of OXA-48 resistance gene is considered to be an endemic problem in Turkey. Although the number of isolates were limited in the current study, our results indicate that the predominant mechanism of carbapenemase resistance is indeed OXA-48 presence.

Several phenotypic confirmation methods have been developed to detect carbapenemase. Previous studies have utilized high levels of temocillin resistance as a diagnostic marker for the presence of OXA-48 carbapenemase. In an investigation of 2280 multiresistant E.coli, Klebsiella spp. and Enterobacter spp. isolates, Woodford et al. found that high temocillin resistance $(\mathrm{MIC}>128 \mathrm{mg} / \mathrm{L})$ was present in 98 of the 108 (90.7\%) OXA-48-positive strains [17]. When they assessed for diagnostic accuracy, they reported a very low PPV (\%27.6) with high NPV (\%99.5) for diagnostic capability. Another study, by Shaker et al. revealed in 48 OXA-48-positive CPE isolates that $30 \mu \mathrm{g}$ temocillin disc had 97.9\% sensitivity, 50\% specificity, 97.95\% PPV and 50\% NPV for detecting OXA-48 production [18]. We demonstrated that temocillin disk diffusion method had a sensitivity of $100 \%$ and a specificity of $77.8 \%$ in detecting the presence of OXA-48. This result taken together with prior publications indicates that high-level resistance of temocillin is insufficient as the only criterion for determining OXA-48 
type resistance. However, our results support the notion that OXA-48 positivity can be mostly excluded in the absence of high-level temocillin resistance, and temocillin can be used as a part of the algorithm rather than as a single test that can be used in carbapenemase identification.

Although molecular tests are the gold standard for identifying the presence of genes encoding OXA-48 carbapenemase groups, they are time-consuming and may not be affordable for laboratories due to high reagent costs and the need for specialized equipment and/or trained personnel. As an alternative method, immunochromatographic tests have been proposed as a method for their identification of OXA-48 carbapenemase directly from suspected colonies grown on primary plates [19]. These tests are based on the principle that the complex structure formed (as a result of the combination of monoclonal antibodies bound to a nitrocellulose membrane with antigen present in the sample) causes color change in less than 15 minutes [19]. Rubio et al. demonstrated in 37 isolates expressing OXA-48 carbapenemase that the OXA-48 K-set test had $100 \%$ sensitivity and specificity for detecting OXA-48 carbapenemase [20]. Koroska et al. showed in 166 Enterobacteriaceae isolates, including isolates producing OXA -48 carbapenemase $(n=84)$ or Ambler class A and B carbapenemase $(n=41)$ and carbapenemase-negative isolates $(n=41)$ that the sensitivity and specificity were $100 \%$ and $43.9 \%$ for temocillin disc diffusion test, while they were $100 \%$ and $100 \%$ for the OXA-48 K-Set test, respectively [21]. Wareham et al. found in 82 Enterobacteriaceae isolates that 53 isolates produced OXA-48-like carbapenemase via molecular analysis, and the OXA-48 $\mathrm{K}$-SeT assay gave a positive result for all 53 OXA-48-producing strains and was negative for all of the other isolates tested [22]. In accordance with these studies, we found $100 \%$ specificity and sensitivity with the OXA-48 K-Set test when evaluated according to RTPCR results. The OXA-48 K-Set had the highest sensitivity and specificity among the tests that can be used in the identification of OXA-48 type carbapenemase, and seems to be an excellent test in areas with high prevalence of OXA-48like carbapenemases or in epidemic situations where rapid results are imperative. The test is easy to perform and results can be obtained within 15 minutes. However, the OXA-48 KSet is more expensive than simple disk tests and thus may not be an ideal test in areas with a high prevalence of other carbapenemases, including KPC and NDM.

Chromogenic culture media are also useful for the detection of carbapenem-resistant bacteria, and studies with these media have been published. However, studies evaluating OXA-48 carbapenemase-producing pathogens using chromogenic media are limited. Girlich et al. showed in a comparative study with different chromogenic media including 117 Enterobacteriaceae isolates that 53 were OXA48-positive and ChromID media had the highest sensitivity (91\%) and specificity (100) for the detection of OXA-48- producing Enterobacteriaceae -compared to SUPERCARBA and chromID CARBA media [23]. A comparative study with chromID OXA-48 agar and chromID CARBA including 302 rectal swab samples found that 33 isolates had OXA-48 positivity and the sensitivity and specificity of the detection of CPE was $75.8 \%$ and $99.3 \%$ for chromID OXA-48 agar, $57.6 \%$ and $98.9 \%$ for chromID CARBA [24]. Recently, Genc et al. performed a comparative study including $100 \mathrm{~K}$. pneumonia isolates assessed with the OXA-48 K-Set test and four chromogenic media (CHROMagar KPC, CHROMagar mSuperCARBA, ChromID Carba and ChromID OXA-48). The authors found that 60 of the isolates were positive for OXA-48, and reported that the sensitivities and specificities of all chromogenic media (and the rapid test) were 100\% [9]. In accordance with these studies, we found that the ChromID OXA-48 media had a sensitivity of $100 \%$ and a specificity of $88.8 \%$ for detection of OXA-48 positive K. pneumoniae isolates. Our result indicates that ChromID media is a sensitive tool for detection of OXA-48 positivity, but has lower specificity compared to the OXA-48 K-set test. We believe that ChromID media can be used as a form of follow-up testing with great success.

As mentioned before, the primary limitation of this study is that a relatively small number of isolates were evaluated. Secondly, we assessed only OXA-48 carbapenemase in $\mathrm{K}$. pneumonia isolates and did not include KPC or NDM. It is critical to note that the inclusion of other isolates could influence the accuracy of each test in a different manner.

\section{CONCLUSION}

The OXA-48 K-Set test had excellent performance for detecting OXA-48 carbapenemase and can be used easily and reliably in the clinical microbiology routine laboratory for the rapid detection of OXA-48-producing K. pneumoniae isolates, particularly in endemic regions. Also, ChromID media and temocillin disc diffusion testing can be applied in laboratories as a part of the identification and screening algorithms of carbapenemase producing Klebsiella pneumonia; however, the limitations must be considered and these tests cannot be seen as stand-alone alternatives to RT-PCR.

$\begin{array}{ll} & \text { ABBREVIATIONS } \\ \text { PCR: } & \text { Polymerase chain reaction; } \\ \text { TDD: } & \text { temocillin disc diffusion } \\ \text { K. pneumoniae: } & \text { Klebsiella pneumoniae } \\ \text { CR-Kp: } & \text { carbapenem-resistant K. pneumoniae } \\ \text { KPC: } & \text { Klebsiella pneumoniae carbapenemase } \\ \text { NDM: } & \text { New Delhi metallo-b-lactamase } \\ \text { OXA: } & \text { oxacillinase } \\ \text { RT-PCR: } & \text { real-time PCR } \\ \text { PPV: } & \text { positive predictive value } \\ \text { NPV: } & \text { negative predictive value }\end{array}$


Author contributions: All authors have sufficiently contributed to the study, and agreed with the results and conclusions.

Funding: No funding source is reported for this study.

Declaration of interest: No conflict of interest is declared by authors.

\section{REFERENCES}

1. Choby JE, Howard-Anderson J, Weiss DS Hypervirulent Klebsiella pneumoniae-clinical and molecular perspectives. J Intern Med 2020; 287(3): 283300. doi: 10.1111/joim.13007.

2. Logan LK, Weinstein RA. The epidemiology of carbapenem-resistant Enterobacteriaceae: the impact and evolution of a global menace. J Infect Dis 2017; 215(suppl_1): S28-S36. doi: 10.1093/infdis/jiw282.

3. Tacconelli E, Carrara E, Savoldi A, et al. Discovery, research, and development of new antibiotics: the WHO priority list of antibiotic-resistant bacteria and tuberculosis. Lancet Infect Dis 2018; 18(3): 318-27. doi: 10.1016/S1473-3099(17)30753-3.

4. Zarakolu P, Eser OK, Aladag E, et al. Epidemiology of carbapenem-resistant Klebsiella pneumoniae colonization: a surveillance study at a Turkish university hospital from 2009 to 2013. Diagn Microbiol Infect Dis 2016; 85(4): 466-70. doi: 10.1016/j.diagmicrobio.2016.05.012.

5. Chen C-M, Guo M-K, Ke S-C, et al. Emergence and nosocomial spread of ST11 carbapenem-resistant Klebsiella pneumoniae co-producing OXA-48 and KPC2 in a regional hospital in Taiwan. J Med Microbiol 2018; 67(7): 957-64. doi: 10.1099/jmm.0.000771.

6. Poirel L, Héritier C, Tolün V, Nordmann P. Emergence of oxacillinase-mediated resistance to imipenem in Klebsiella pneumoniae. Antimicrob Agents Chemother 2004; 48(1): 15-22. doi: 10.1128/AAC.48.1.15-22.2004.

7. Nordmann P, Gniadkowski M, Giske C, et al. Identification and screening of carbapenemaseproducing Enterobacteriaceae. Clin Microbiol Infect 2012; 18(5): 432-8. doi: 10.1111/j.14690691.2012.03815.x.

8. Sağıroğlu P, Hasdemir U, Gelmez GA, Aksu B, Karatuna O, Söyletir G. Performance of "RESIST-3 OKN K-SeT" immunochromatographic assay for the detection of OXA-48 like, KPC, and NDM carbapenemases in Klebsiella pneumoniae in Turkey. Braz J Microbiol 2018; 49(4): 885-90. doi: 10.1016/j.bjm.2018.02.002.

9. Genc O, Aksu E. Chromogenic culture media or rapid immunochromatographic test: which is better for detecting Klebsiella pneumoniae that produce OXA-48 and can they be used in blood and urine specimens. J Microbiol Methods 2018; 148: 169-73. doi: 10.1016/j.mimet.2018.04.014.
10. Meng X, Yang J, Duan J, et al. Assessing molecular epidemiology of carbapenem-resistant Klebsiella pneumoniae (CR-KP) with MLST and MALDI-TOF in Central China. Sci Rep 2019; 9(1): 1-9. doi: 10.1038/s41598-018-38295-8.

11. Livorsi DJ, Chorazy ML, Schweizer ML, et al. A systematic review of the epidemiology of carbapenemresistant Enterobacteriaceae in the United States. Antimicrob Resist Infect Control 2018; 7(1): 55. doi: 10.1186/s13756-018-0346-9.

12. Grundmann H, Glasner C, Albiger B, et al. Occurrence of carbapenemase-producing Klebsiella pneumoniae and Escherichia coli in the European survey of carbapenemase-producing Enterobacteriaceae (EuSCAPE): a prospective, multinational study. Lancet Infect Dis 2017; 17(2): 153-63. doi: 10.1016/S14733099(16)30257-2.

13. Albiger B, Glasner C, Struelens MJ, Grundmann H, Monnet DL. Carbapenemase-producing Enterobacteriaceae in Europe: assessment by national experts from 38 countries, May 2015. Eurosurveillance 2015; 20(45): 30062. doi: 10.2807/15607917.ES.2015.20.45.30062.

14. Brolund A, Lagerqvist N, Byfors S, et al. Worsening epidemiological situation of carbapenemase-producing Enterobacteriaceae in Europe, assessment by national experts from 37 countries, July 2018. Eurosurveillance 2019; 24(9): 1900123. doi: 10.2807/15607917.ES.2019.24.9.1900123.

15. Glasner C, Albiger B, Buist G, et al. Carbapenemaseproducing Enterobacteriaceae in Europe: a survey among national experts from 39 countries, February 2013. Eurosurveillance 2013; 18(28): 20525. doi: 10.2807/1560-7917.ES2013.18.28.20525.

16. Davarci I, Senbayrak S, Aksaray S, et al. Molecular Epidemiology of Carbapenemresistant Klebsiella pneumoniae Isolates. Anatolian Clinic 2019; 24(1): 1-7. doi: 10.21673/anadoluklin.423081.

17. Woodford N, Pike R, Meunier D, Loy R, Hill R, Hopkins $\mathrm{KL}$. In vitro activity of temocillin against multidrugresistant clinical isolates of Escherichia coli, Klebsiella spp. and Enterobacter spp., and evaluation of high-level temocillin resistance as a diagnostic marker for OXA-48 carbapenemase. J Antimicrob Chemother 2014; 69(2): 564-7. doi: 10.1093/jac/dkt383.

18. Shaker OA, Gomaa HE, Elmasry SA, Halim RMA, Abdelrahman AH, Kamal JS. Evaluation of Combined Use of Temocillin Disk and Mastdisks Inhibitor Combination Set Against Polymerase Chain Reaction for Detection of Carbapenem-Resistant Enterobacteriaceae. Open Access Maced J Med Sci 2018; 6(2): 242-7. doi: 10.3889/oamjms.2018.090. 
19. Kolenda C, Benoit R, Carricajo A, Bonnet R, Dauwalder $O$, Laurent F. Evaluation of the new multiplex immunochromatographic OKNV K-Set assay for the rapid detection of OXA-48-like, KPC, NDM and VIM carbapenemases. J Clin Microbiol 2018; 56(11): e0124718. doi: 10.1128/JCM.01247-18.

20. Rubio E, Zboromyrska Y, Pitart C, et al. Evaluation of a rapid immunochromatographic test for the detection of OXA-48 carbapenemase. Diagn Microbiol Infect Dis 2017; 87(3): 266-7. doi: 10.1016/j.diagmicrobio.2016.12.001.

21. Koroska F, Göttig S, Kaase M, et al. Comparison of phenotypic tests and an immunochromatographic assay and development of a new algorithm for detection of OXA-48-like carbapenemases. J Clin Microbiol 2017; 55(3): 877-83. doi: 10.1128/JCM.01929-16.
22. Wareham DW, Shah R, Betts JW, Phee LM, Momin MHFA. Evaluation of an immunochromatographic lateral flow assay (OXA-48 K-SeT) for rapid detection of OXA-48-like carbapenemases in Enterobacteriaceae. J Clin Microbiol 2016; 54(2): 471-3. doi: 10.1128/JCM.02900-15.

23. Girlich D, Anglade C, Zambardi G, Nordmann P. Comparative evaluation of a novel chromogenic medium (chromID OXA-48) for detection of OXA-48 producing Enterobacteriaceae. Diagn Microbiol Infect Dis 2013; 77(4): 296-300. doi: 10.1016/j.diagmicrobio.2013.08.015.

24. Zarakolu P, Day K, Sidjabat H, et al. Evaluation of a new chromogenic medium, chromID OXA-48, for recovery of carbapenemase-producing Enterobacteriaceae from patients at a university hospital in Turkey. Eur J Clin Microbiol Infect Dis 2015; 34(3): 519-25. doi: 10.1007/s10096-014-2255-z. 